Geometry \& Topology

Volume 3 (1999) 103-118

Published: 28 May 1999

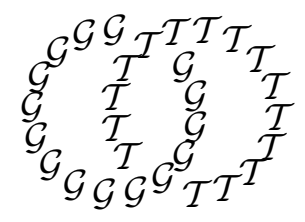

\title{
Embeddings from the point of view of immersion theory : Part II
}

\author{
Thomas G Goodwillie \\ MiCHAEL WEISS \\ Department of Mathematics, Brown University \\ Box 1917, Providence RI 02912-0001, USA \\ and \\ Department of Mathematics, University of Aberdeen \\ Aberdeen AB24 3UE, UK
}

Email: tomg@math.brown.edu and m.weiss@maths.abdn.ac.uk

\begin{abstract}
Let $M$ and $N$ be smooth manifolds. For an open $V \subset M$ let $\operatorname{emb}(V, N)$ be the space of embeddings from $V$ to $N$. By the results of Goodwillie [4], [5], [6] and Goodwillie-Klein [7], the cofunctor $V \mapsto \operatorname{emb}(V, N)$ is analytic if $\operatorname{dim}(N)-\operatorname{dim}(M) \geq 3$. We deduce that its Taylor series converges to it. For details about the Taylor series, see Part I (reference [15]).
\end{abstract}

AMS Classification numbers Primary: 57R40

Secondary: 57R42

Keywords: Embedding, immersion, calculus of functors

Proposed: Ralph Cohen

Seconded: Haynes Miller, Gunnar Carlsson
Received: 10 May 1998

Revised: 5 May 1999

Copyright Geometry and Topology 


\section{Introduction}

This is a continuation of [15]. The ideas in this second part are mostly due to Goodwillie; notes and illustrations mostly by Weiss. For introductions and notation generally speaking, see [14] and [15]. We fix smooth manifolds $M^{m}$ and $N^{n}$, without boundary unless otherwise stated, and we assume $m \leq n$. As in [15] we write $\mathcal{O}$ for the poset of open subsets of $M$. Then $V \mapsto \operatorname{emb}(V, N)$ is a cofunctor from $\mathcal{O}$ to Spaces (= fibrant simplicial sets), and from [14] and [15] we have a fairly thorough understanding of its Taylor approximations, the cofunctors $V \mapsto T_{k} \operatorname{emb}(V, N)$. Here we show that the Taylor approximations are good approximations. The main result is Corollary 2.5. Sections 4 and 5 contain illustrations.

Following are some conventions.

Let $R$ be a set with $r$ elements. A functor $\mathcal{X}$ from the poset $\mathcal{P}_{R}$ of subsets of $R$ to Spaces is an $r$-dimensional cube of Spaces [3, section 1]. The cube is $k-$ Cartesian if the canonical map

$$
\mathcal{X}(\emptyset) \longrightarrow \operatorname{holim}_{\{S \mid \emptyset \neq S \subset R\}} \mathcal{X}(S)
$$

is $k$-connected. A Cartesian cube is one which is $k$-Cartesian for all $k$. Since $\mathcal{P}_{R}$ as a category is isomorphic to its own opposite, we can use similar terminology for cofunctors from $\mathcal{P}_{R}$ to Spaces. Such a cofunctor will also be called a cube. It is $k$-Cartesian if the canonical map

$$
\mathcal{X}(R) \longrightarrow \operatorname{holim}_{\{S \mid S \subset R, S \neq R\}} \mathcal{X}(S)
$$

is $k$-connected, and Cartesian if it is $k$-Cartesian for all $k$.

Let $\mathbb{R}_{\llcorner}^{n+2}:=[0, \infty) \times[0, \infty) \times \mathbb{R}^{n}$. An $(n+2)$-dimensional smooth manifold triad is a paracompact Hausdorff space $Q$ together with a maximal atlas consisting of open subsets $V_{i} \subset Q$ and open embeddings $\phi_{i}: V_{i} \rightarrow \mathbb{R}_{\llcorner}^{n+2}$ satisfying the following conditions:

- The union of the $V_{i}$ is $Q$.

- The changes of charts $\phi_{i}\left(\phi_{j}\right)^{-1}$ are smooth where defined, and take points in $\mathbb{R}_{\llcorner}^{n+2}$ with vanishing first coordinate (vanishing second coordinate) to points with vanishing first coordinate (vanishing second coordinate).

Let $\partial_{0} Q$ be the set of those $x \in Q$ which under some chart $\phi_{i}$ map to a point in $\mathbb{R}_{\llcorner}^{n+2}$ with vanishing first coordinate. Also, let $\partial_{1} Q$ be the set of those $x \in Q$ which under some chart $\phi_{i}$ map to a point in $\mathbb{R}_{\llcorner}^{n+2}$ with vanishing second coordinate. Then $Q$ is an $(n+2)$-dimensional smooth manifold with corners; its boundary is $\partial_{0} Q \cup \partial_{1} Q$ and its corner set is $\partial_{0} Q \cap \partial_{1} Q$. 
The handle index of the manifold triad $Q$ is the smallest integer $a$ such that $Q$ can be built from a collar on $\partial_{0} Q$ by attaching handles of index $\leq a$. (It may happen that $Q$ is a collar on $\partial_{0} Q$. When that is the case we say that the handle index is $-\infty$.)

Example Suppose that $P$ is smooth, with boundary, and let $f: P \rightarrow \mathbb{R}$ be smooth. If 0 is a regular value both for $f$ and for $f \mid \partial P$, then the inverse image of $[0, \infty)$ under $f$ is a manifold triad $Q$, with $\partial_{0} Q=\partial P \cap Q$. (If $\partial P=\emptyset$, then of course $\partial_{0} Q=\emptyset$.) Any $Q \subset P$ which can be obtained in this way will be called a codimension zero subobject of $P$.

\section{Excision Estimates}

Let $Y$ be a smooth $n$-manifold with boundary. Let $Q_{0}, Q_{1}, \ldots, Q_{r}$ be smooth compact $n$-manifold triads with handle index $q_{i}$ where $n-q_{i} \geq 3$. Suppose that smooth embeddings $e_{i}: \partial_{0} Q_{i} \rightarrow \partial Y$ have been selected for $0 \leq i \leq r$, and that their images are pairwise disjoint. For $S \subset[r]=\{0, \ldots, r\}$ let $Q_{S}$ be the disjoint union of the $Q_{i}$ with $i \in S$. By $\operatorname{emb}\left(Q_{S}, Y\right)$ we mean the Space of smooth embeddings $f: Q_{S} \rightarrow Y$ which satisfy $f \mid \partial_{0} Q_{i}=e_{i}$ and $f^{-1}(\partial Y)=\partial_{0} Q_{S}$.

1.1 Theorem ([4], [5], [6], [7]) The $(r+1)$-cube taking a subset $S$ of $[r]$ to $\operatorname{emb}\left(Q_{S}, Y\right)$ is $\left(3-n+\sum_{i=0}^{r}\left(n-q_{i}-2\right)\right)$-Cartesian, provided $r \geq 1$.

Comment Assuming that $\operatorname{emb}\left(Q_{[r]}, Y\right)$ is nonempty, fix a base point $f$ in $\operatorname{emb}\left(Q_{[r]}, Y\right)$. Let the image of $f$ in $\operatorname{emb}\left(Q_{S}, Y\right)$ serve as base point for $\operatorname{emb}\left(Q_{S}, Y\right)$. Goodwillie shows in [6] that the cube $S \mapsto \Omega \operatorname{emb}\left(Q_{S}, Y\right)$ is $\left(2-n+\sum_{i=0}^{r}\left(n-q_{i}-2\right)\right)-$ Cartesian, provided $r \geq 1$. The delooped statement, Theorem 1.1 just above, has been proved by Goodwillie and Klein and will appear in [7].

We will need a slight generalization of 1.1 where the $Q_{i}$ are allowed to have dimension $m \leq n$. For this and other purposes we need a lemma.

1.2 Lemma Let $u: \mathcal{X} \rightarrow \mathcal{Y}$ be a map of $(r+1)$-cubes. That is, $\mathcal{X}$ and $\mathcal{Y}$ are functors from the poset of subsets of $[r]$ to Spaces, and $u$ is a natural transformation. Suppose that $\mathcal{Y}$ is $k$-Cartesian and, for every $y \in \mathcal{Y}(\emptyset)$, the $(r+1)$-cube defined by

$$
S \mapsto \operatorname{hofiber}[\mathcal{X}(S) \stackrel{u}{\rightarrow} \mathcal{Y}(S)]
$$

is $k$-Cartesian. (The homotopy fiber is taken over the image of $y$ in $\mathcal{Y}(S)$.) Then $\mathcal{X}$ is $k$-Cartesian. 
Proof of 1.2 Combine $[3,1.18]$ with $[3,1.6]$.

Now let $Y$ and $Q_{i}$ be as in 1.1, assuming however $\operatorname{dim}\left(Q_{i}\right)=m \leq n$. As before, embeddings $e_{i}: \partial_{0} Q_{i} \rightarrow \partial Y$ are specified and $n-q_{i} \geq 3$, where $q_{i}$ is the handle index of $Q_{i}$. We want to show that the conclusion of 1.1 still holds. Without loss of generality, $Y$ is a smooth submanifold of some $\mathbb{R}^{t}$. Then we can define maps

$$
\operatorname{emb}\left(Q_{S}, Y\right) \longrightarrow \operatorname{map}\left(Q_{S}, G_{n-m}\right) \quad ; \quad f \mapsto \nu_{f}
$$

where $G_{n-m}$ is the direct limit of the Grassmannians of $(n-m)$-dimensional linear subspaces of $\mathbb{R}^{u}$, for $u \geq 0$, and where $\nu_{f}$ takes $x \in Q_{S}$ to the intersection of the tangent space of $Y$ at $f(x)$ with the normal space of $f\left(Q_{S}\right)$ at $f(x)$. In other words, $\nu_{f}$ classifies the normal bundle of the embedding $f$. We have therefore a map of $(r+1)$-cubes:

$$
\left\{S \mapsto \operatorname{emb}\left(Q_{S}, Y\right)\right\} \longrightarrow\left\{S \mapsto \operatorname{map}\left(Q_{S}, G_{n-m}\right)\right\} .
$$

Since the codomain cube in $(\bullet)$ is Cartesian, the contravariant analog of 1.2 tells us that the domain cube is $k$-Cartesian provided that, for every $\xi$ in $\operatorname{map}\left(Q_{[r]}, G_{n-m}\right)$, the cube of homotopy fibers

$$
\left.(\bullet \bullet) \quad S \mapsto \operatorname{hofiber}_{\xi}\left[\operatorname{emb}\left(Q_{S}, Y\right)\right\} \longrightarrow \operatorname{map}\left(Q_{S}, G_{n-m}\right)\right]
$$

is $k$-Cartesian. Now it is easy to construct a homotopy equivalence from the homotopy fiber of $\operatorname{emb}\left(Q_{S}, Y\right) \rightarrow \operatorname{map}\left(Q_{S}, G_{n-m}\right)$ to $\operatorname{emb}\left(Q_{S}^{\prime}, Y\right)$ where $Q_{S}^{\prime}$ is the total space of the disk bundle on $Q_{S}$ determined by $\xi$. This construction is natural in $S$, so $(\bullet \bullet)$ is $k$-Cartesian if the cube $S \mapsto \operatorname{emb}\left(Q_{S}^{\prime}, Y\right)$ is $k_{-}^{-}$ Cartesian, which by 1.1 is the case if $k=\left(3-n+\sum_{i=0}^{r}\left(n-q_{i}-2\right)\right)$. Therefore:

1.3 Observation Theorem 1.1 generalizes to the situation where the $Q_{i}$ have dimension $m \leq n$.

The corollary below is a technical statement about the cofunctor on $\mathcal{O}$ given by $V \mapsto \operatorname{emb}(V, N)$. Suppose that $P$ is a smooth compact codimension zero subobject of $M$, and that $Q_{0}, \ldots, Q_{r}$ are pairwise disjoint compact codimension zero subobjects of $M \backslash \operatorname{int}(P)$. For $S \subset\{0, \ldots, r\}=[r]$ let $V_{S}$ be the interior of $P \cup Q_{S}$ where $Q_{S}$ is the union of the $Q_{i}$ for $i \in S$. We write $V$ instead of $V_{\emptyset}$. Suppose that $Q_{i}$ has handle index $q_{i} \leq n-3$.

1.4 Corollary Assume $r \geq 1$. The $(r+1)$-cube taking a subset $S$ of $[r]$ to $\operatorname{emb}\left(V_{S}, N\right)$ is $\left(3-n+\sum_{i=0}^{r}\left(n-q_{i}-2\right)\right)$-Cartesian. 
Proof Let $\bar{V}_{S}$ be the closure of $V_{S}$ in $M$. Let $\operatorname{emb}\left(\bar{V}_{S}, N\right)$ be the inverse limit of the Spaces (simplicial sets) emb $(U, N)$ where $U$ ranges over the neighborhoods of $V_{S}$ in $M$. The restriction from $\operatorname{emb}\left(\bar{V}_{S}, N\right)$ to $\operatorname{emb}\left(V_{S}, N\right)$ is a homotopy equivalence. Using this fact and 1.2, we see that it is enough to show that for every embedding $f: \bar{V} \rightarrow N$, the $(r+1)$-cube

$$
S \mapsto \operatorname{hofiber}\left[\operatorname{emb}\left(\bar{V}_{S}, N\right) \stackrel{\text { res }}{\longrightarrow} \operatorname{emb}(\bar{V}, N)\right]
$$

is $\left(3-n+\Sigma_{i=0}^{r}\left(n-q_{i}-2\right)\right)$-Cartesian. The homotopy fibers are to be taken over the point $f$ in $\operatorname{emb}(\bar{V}, N)$, which we fix for the rest of this proof. By the isotopy extension theorem, the restriction maps from $\operatorname{emb}\left(\bar{V}_{S}, N\right)$ to $\operatorname{emb}(\bar{V}, N)$ are Kan fibrations. Therefore it is enough to show that

$$
S \mapsto \operatorname{fiber}\left[\operatorname{emb}\left(\bar{V}_{S}, N\right) \stackrel{\text { res }}{\longrightarrow} \operatorname{emb}(\bar{V}, N)\right]
$$

is a $\left(3-n+\sum_{i=0}^{r}\left(n-q_{i}-2\right)\right)$-Cartesian $(r+1)$-cube. Let $D(\bar{V})$ be the total space of a normal disk bundle for $\bar{V}$ in $N$, with corners rounded off, so that $D(\bar{V})$ is a smooth codimension zero subobject of $N$. Let $Y$ be the closure of $N \backslash D(\bar{V})$ in $N$. Let $\mathcal{X}(S) \subset \operatorname{emb}\left(Q_{S}, Y\right)$ be the Space of embeddings $g: Q_{S} \rightarrow Y$ for which the map $f \cup g$ from $\bar{V}_{S}=\bar{V} \cup Q_{S}$ to $N$ is smooth. (Here all embeddings from $Q_{S}$ to $Y$ are prescribed on $\partial_{0} Q_{S}$, as in 1.1 and 1.3.) The inclusions of $\mathcal{X}(S)$ in $\operatorname{emb}\left(Q_{S}, Y\right)$ and the fiber of the restriction map $\operatorname{emb}\left(\bar{V}_{S}, N\right) \rightarrow \operatorname{emb}(\bar{V}, N)$ are homotopy equivalences. Therefore it is enough to know that $S \mapsto \operatorname{emb}\left(Q_{S}, Y\right)$ is $\left(3-n+\sum_{i=0}^{r}\left(n-q_{i}-2\right)\right)$-Cartesian, which we know from 1.3.

\section{Convergence}

We begin with an abstraction. Suppose that $G$ is a good cofunctor from $\mathcal{O}$ to Spaces [14, 2.2]. Fix an integer $\rho>0$. Let $P$ be a smooth compact codimension zero subobject of $M$, and let $Q_{0}, \ldots, Q_{r}$ be pairwise disjoint compact codimension zero subobjects of $M \backslash \operatorname{int}(P)$. Suppose that $Q_{i}$ has handle index $q_{i}<\rho$. Let $V_{S}=\operatorname{int}\left(P \cup Q_{S}\right)$ as in 1.4. Assume also $r \geq 1$.

2.1 Definition The cofunctor $G$ is $\rho$-analytic with excess $c$ if, in these circumstances, the $(r+1)$-cube $S \mapsto G\left(V_{S}\right)$ is $\left(c+\Sigma_{i=0}^{r}\left(\rho-q_{i}\right)\right)$-Cartesian.

2.2 Example According to 1.4, the cofunctor $V \mapsto \operatorname{emb}(V, N)$ is $(n-2)-$ analytic, with excess $3-n$.

2.3 Theorem Suppose that $G$ is $\rho$-analytic with excess $c$, and that $W \in \mathcal{O}$ has a proper Morse function whose critical points are all of index $\leq q$, where $q<\rho$. Then $\eta_{k-1}: G(W) \rightarrow T_{k-1} G(W)$ is $(c+k(\rho-q))$-connected, for any $k>1$. 
Proof With a homotopy inverse limit argument we can reduce to the case where $W$ is tame (ie, is the interior of a compact codimension zero subobject of $M$ ) and $\bar{W}$ has a smooth handle decomposition with handles of index $\leq q$ only.

Case $1: q=0$ Then $W$ is the union of disjoint open $m$-balls $W_{i}$ with $1 \leq i \leq \ell$ for some $\ell$. For $S \subset\{1, \ldots, \ell\}$ we write $W_{S}=\cup_{i \in S} W_{i}$. If $\ell<k$ then $\eta_{k-1}$ from $G(W)$ to $T_{k-1} G(W)$ is a homotopy equivalence by definition of $T_{k-1} G$. Assume therefore $\ell \geq k$. The diagram

$$
T_{\ell} G(W) \stackrel{r_{\ell}}{\longrightarrow} T_{\ell-1} G(W) \stackrel{r_{\ell-1}}{\longrightarrow} \cdots \stackrel{r_{k}}{\longrightarrow} T_{k-1} G(W)
$$

can be identified up to homotopy equivalences with

$$
J_{\ell} \rightarrow J_{\ell-1} \rightarrow \cdots \rightarrow J_{k} \rightarrow J_{k-1}
$$

where $J_{t}$ is the homotopy inverse limit of the $G\left(W_{S}\right)$ with $S \subset\{1, \ldots, \ell\}$ and $|S| \leq t$. (See also the last lines of the proof of $[15,9.1]$.) The fibration $J_{t} \rightarrow J_{t-1}$ is obtained by pullback from another fibration, namely the product over all $S \subset\{1, \ldots, \ell\}$ with $|S|=t$ of the fibration

$$
p_{S}: \operatorname{holim}_{\{R \mid R \subset S\}} G\left(W_{R}\right) \longrightarrow \operatorname{holim}_{\{R \mid R \subset S, R \neq S\}} G\left(W_{R}\right) .
$$

All this is true without any special assumptions on $G$ except goodness. But now we use the analyticity hypothesis and find that $p_{S}$ is $(c+t \rho)$-connected where $t=|S|$. Therefore the composition

$$
r_{k} r_{k+1} \ldots r_{\ell}: T_{\ell} G(W) \rightarrow T_{k-1} G(W)
$$

is $(c+k \rho)$-connected. We can identify it with $\eta_{k-1}: G(W) \rightarrow T_{k-1} G(W)$ since $\eta_{\ell}$ from $G(W)$ to $T_{\ell} G(W)$ is a homotopy equivalence.

Case 2 : $q>0$ We induct on $q$. For every $q$-handle $Q_{u}$ in the handle decomposition of $\bar{W}$ choose a smooth chart $\phi_{u}: Q_{u} \cong \mathbb{D}^{q} \times \mathbb{D}^{m-q}$ and distinct points $x_{u, i}$ in the interior of $\mathbb{D}^{q}$ for $1 \leq i \leq k$. Let $A_{u, i} \subset W$ be the inverse image of $\left\{x_{u, i}\right\} \times \operatorname{int}\left(\mathbb{D}^{m-q}\right)$ under $\phi_{u}$. Each $A_{u, i}$ is a closed smooth codimension $q$ submanifold of $W$, meeting the core of handle $Q_{u}$ transversely in one point. For $S \subset\{1, \ldots, k\}$ let $A_{S}$ be the union of all $A_{u, i}$ with $i \in S$ and $u$ arbitrary. Our analyticity assumption on $G$ implies that the cube defined by $S \mapsto G\left(W \backslash A_{S}\right)$ is $(c+k(\rho-q))$-Cartesian. The cube $S \mapsto T_{k-1} G\left(W \backslash A_{S}\right)$ is Cartesian $\left(=\infty\right.$-Cartesian). For each nonempty $S$, the manifold $W \backslash A_{S}$ has an isotopy equivalent tame codimension zero submanifold with a handle decomposition where all handles have indices $<q$. Therefore by inductive assumption, the map $\eta_{k-1}: G\left(W \backslash A_{S}\right) \rightarrow T_{k-1} G\left(W \backslash A_{S}\right)$ is $(c+k(\rho-q+1))$-connected, provided $S \neq \emptyset$. It follows with $[3,1.22]$ that $\eta_{k-1}$ induces a map

$$
\operatorname{holim} G\left(W \backslash A_{S}\right) \longrightarrow \operatorname{holim} T_{k-1} G\left(W \backslash A_{S}\right)
$$


which is $(c+k(\rho-q+1)-k+1)$-connected (both homotopy inverse limits are over nonempty $S \subset\{1, \ldots, k\})$. Combining this with our "Cartesianness" estimates for the cubes $S \mapsto G\left(W \backslash A_{S}\right)$ and $S \mapsto T_{k-1} G\left(W \backslash A_{S}\right)$, where $S$ again denotes an arbitrary subset of $\{1, \ldots, k\}$, we can conclude that $G(W) \rightarrow T_{k-1} G(W)$ is indeed $(c+k(\rho-q))$-connected.

2.4 Corollary Suppose that $G$ is $\rho$-analytic. If $\rho>m$, then the canonical map $G(W) \longrightarrow \operatorname{holim}_{k} T_{k} G(W)$ is a homotopy equivalence for every $W$ in $\mathcal{O}$. In general, the map $G(W) \rightarrow \operatorname{holim}_{k} T_{k} G(W)$ is a homotopy equivalence if $W$ has a proper Morse function whose critical points are all of index $<\rho$.

2.5 Corollary Let $G(W)=\operatorname{emb}\left(W, N^{n}\right)$ for open $W \subset M^{m}$. If $m$ is less than $n-2$, then $G(W) \simeq \operatorname{holim}_{k} T_{k} G(W)$ for all $W$. If $m=n-2$, then $G(W) \simeq \operatorname{holim}_{k} T_{k} G(W)$ provided $W$ has no compact component. In general: suppose that $W$ has a proper Morse function whose critical points are all of index $\leq q$, where $q<n-2$. Then $\eta_{k}: G(W) \rightarrow T_{k} G(W)$ is $(k(n-2-q)-q+1)-$ connected for $k \geq 1$. Consequently $G(W) \simeq \operatorname{holim}_{k} T_{k} G(W)$.

Proof This follows from 2.4, 2.3 and 2.2 with $\rho=n-2$ and $c=3-n$.

2.6 Corollary Let $f: G_{1} \rightarrow G_{2}$ be a natural transformation between good cofunctors on $\mathcal{O}$. Suppose that $G_{1}$ and $G_{2}$ are both $\rho$-analytic and $f$ from $G_{1}(W)$ to $G_{2}(W)$ is a homotopy equivalence whenever $W$ is a tubular neighborhood of a finite set $\left(W \in \mathcal{O} j\right.$ for some $j$ ). Then $f: G_{1}(W) \rightarrow G_{2}(W)$ is a homotopy equivalence for any $W$ which has a proper Morse function with critical points of index $<\rho$ only.

Proof The hypothesis on $f$ implies that $T_{k} f: T_{k} G_{1} \rightarrow T_{k} G_{2}$ is an equivalence.

\section{Taylor Approximations of Analytic Cofunctors}

As in the preceding section, $G$ is a good cofunctor from $\mathcal{O}$ to Spaces.

3.1 Proposition Suppose that $G$ is homogeneous of degree $k$ where $k \geq 0$. If $G(V)$ is $(c-1+k \rho)$-connected for every $V$ in $\mathcal{O} k$, and $\rho \geq m$, then $G$ is $\rho$-analytic with excess $c$. 
Remark If $V$ in $\mathcal{O} k$ has $<k$ components, then $G(V)$ is contractible and therefore automatically $(c-1+k \rho)$-connected. The values $G(V)$ for $V$ in $\mathcal{O} k$ with exactly $k$ components can be regarded as the fibers of the classifying (quasi)-fibration for $G$. See [15, section 8] for the classification of homogeneous cofunctors on $\mathcal{O}$.

Proof The case $k=0$ is trivial, so we assume $k \geq 1$. Choose $r$ and $V_{S}$ for $S \subset[r]$ as in 2.1. Again write $V=V_{\emptyset}$. We must show that the cube $S \mapsto G\left(V_{S}\right)$ is a $\left(c+\sum_{i=0}^{r}\left(\rho-q_{i}\right)\right)$-Cartesian $(r+1)$-cube. The case $r \geq k$ is again trivial, so we assume that $r<k$ and proceed by downward induction on $r$. Also the cases where $q_{i}=-\infty$ for some $i$ are trivial, so we assume $q_{i} \geq 0$ for all $i$.

Case $1: q_{i}=0, \forall i$ We do an induction on the number of handles in a handle decomposition of $\bar{V}$. If $\bar{V}$ is empty and $r<k-1$ then $G\left(V_{S}\right)$ is contractible for all $S$, so there is nothing to prove. If $\bar{V}$ is empty and $r=k-1$, then $G\left(V_{S}\right)$ is contractible except possibly when $S=[r]$, in which case it is $(c-1+k \rho)$ connected; then the cube $S \mapsto G\left(V_{S}\right)$ is $(c+k \rho)$-Cartesian, which means, $\left(c+\Sigma\left(\rho-q_{i}\right)\right)$-Cartesian.

For nonempty (but still tame) $\bar{V}$, choose a handle decomposition. If $\bar{B}$ is the cocore of a handle of index $p$ in $\bar{V}$, and $B:=\bar{B} \cap V \cong \mathbb{R}^{m-p}$, then the $(r+2)$-cube

$$
\left\{G\left(V_{S}\right) \mid S \subset[r]\right\} \longrightarrow\left\{G\left(V_{S} \backslash B\right) \mid S \subset[r]\right\}
$$

is $(c+(\rho-p)+(r+1) \rho)$-Cartesian by the downward induction on $r$. The $(r+1)$-subcube $\left\{G\left(V_{S} \backslash B\right) \mid S \subset[r]\right\}$ is $(c+(r+1) \rho)$-Cartesian by the upward induction on the number of handles of $\bar{V}$. Noting that $\rho \geq m \geq p$ and using $[3,1.6]$ we conclude that the cube $\left\{G\left(V_{S}\right) \mid S \subset[r]\right\}$ is also $(c+(r+1) \rho)-$ Cartesian.

Case 2 : $q_{0}>0$ Let $\bar{A}$ and $\bar{B}$ be two parallel but disjoint cocores for the handle $Q_{0} \subset \bar{V}_{[r]}$ and let $\bar{C}$ be a strip between $\bar{A}$ and $\bar{B}$. Let $A=\bar{A} \cap V$, $B=\bar{B} \cap V, C=\bar{C} \cap V$ so that the triad $(C ; A, B)$ is homeomorphic to the triad $\left([0,1] \times \mathbb{R}^{m-q_{0}} ;\{0\} \times \mathbb{R}^{m-q_{0}},\{1\} \times \mathbb{R}^{m-q_{0}}\right)$. Consider the diagram of $r$-cubes

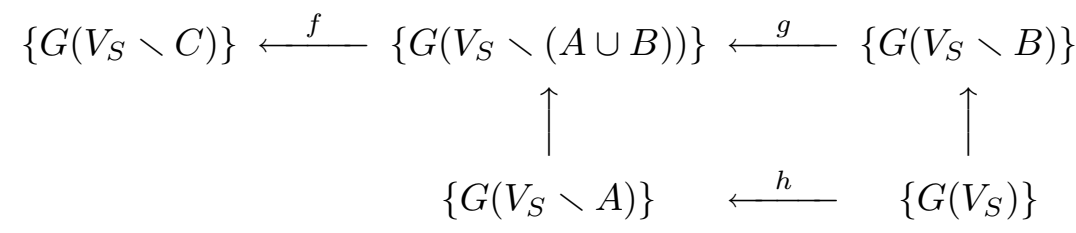

where now $S$ runs through subsets of $[r]$ containing the element 0. (Each arrow in the diagram is a natural transformation of $r$-cubes, induced by appropriate 
inclusions.) We have to show that the arrow $h$ (which is a map of $r$-cubes) is a $\left(c+\Sigma\left(\rho-q_{i}\right)\right)$-Cartesian $(r+1)$-cube. It is enough $[3,1.6]$ to show that $g$ is a $\left(c+\Sigma\left(\rho-q_{i}\right)\right)$-Cartesian $(r+1)$-cube and the square with horizontal arrows $g$ and $h$ is a $\left(c+\Sigma\left(\rho-q_{i}\right)\right)$-Cartesian $(r+2)$-cube. But the composition $f g$ is a Cartesian cube, because for each $S$ containing 0 the inclusion $V_{S} \backslash C \rightarrow V_{S} \backslash B$ is an isotopy equivalence. And $f$ is a $\left(c+1+\Sigma\left(\rho-q_{i}\right)\right)$-Cartesian $(r+1)$-cube by induction on $q_{0}$ (see comments below if this raises doubts). Using [3, 1.8] now, we see that $g$ is indeed a $\left(c+\Sigma\left(\rho-q_{i}\right)\right)$-Cartesian $(r+1)$-cube. What about the square? By the downward induction on $r$ it is a $\left(c+\left(\rho-q_{0}\right)+\Sigma\left(\rho-q_{i}\right)\right)-$ Cartesian $(r+2)$-cube. This is good enough since $\rho \geq m \geq q_{0}$.

Comment Let $\bar{U}$ be the complement of a nice tubular neighborhood of $\bar{A} \cup \bar{B}$ in $\bar{V}_{[r]}$, and $U=\operatorname{int}(\bar{U})$ so that $U$ is tame. Then $\bar{U}$ can be obtained from $\bar{V}$ by attaching $r+1$ handles whose indices are $q_{0}-1, q_{1}, \ldots, q_{r}$. In fact the intersection of $\bar{U}$ with $\bar{C}$ is the cocore of the first handle in the list, with index $q_{0}-1$.

The $(r+1)$-cube determined as in 2.1 by the handle decomposition of $\bar{U}$ relative to $\bar{V}$ can be identified with the cube $f$ above. More precisely, the two cubes are "identified" by a natural transformation from one to the other which is a termwise homotopy equivalence.

3.2 Corollary If $G$ is $\rho$-analytic with excess $c$, then so is $T_{k} G$.

Proof We proceed by induction on $k$. Fix $V$ and $V_{S}$ for $S \subset[r]$ as in 2.1, with $r \geq 0$. We must prove that the $(r+1)$-cube $S \mapsto T_{k} G\left(V_{S}\right)$ is $\left(c+\sum_{i=0}^{r}\left(\rho-q_{i}\right)\right)$ Cartesian. For $k=0$ this is correct.

Suppose $k \geq 1$ and let $z \in T_{k-1} G\left(V_{[r]}\right)$. For open $U \subset V_{[r]}$ let $L_{k}^{z} G(U)$ be the homotopy fiber of the forgetful map $T_{k} G(U) \rightarrow T_{k-1} G(U)$ over the point obtained from $z$ by restriction. The cofunctor $U \mapsto L_{k}^{z} G(U)$ on open subsets $U \subset V_{[r]}$ is homogeneous of degree $k$. If $U \subset V_{[r]}$ is a disjoint union of open $m$-balls, then $L_{k}^{z} G(U)$ is homotopy equivalent to the total homotopy fiber of the cube $Y \mapsto G\left(U_{Y}\right)$ where $Y$ runs over subsets of $\pi_{0}(U)$; see $[15,9.1]$. By the assumption on $G$, this implies that $L_{k}^{z} G(U)$ is $(c+k \rho-1)$-connected. We are now in a position to use 3.1 , and conclude that $L_{k}^{z} G$ is $\rho$-analytic with excess $c$, just like $G$. Now the induction step is easy: we can make a fibration sequence up to homotopy of $(r+1)$-cubes

$$
\left\{L_{k}^{z} G\left(V_{S}\right)\right\} \longrightarrow\left\{T_{k} G\left(V_{S}\right)\right\} \longrightarrow\left\{T_{k-1} G\left(V_{S}\right)\right\} .
$$

By induction, the right-hand cube is $\left(c+\Sigma\left(\rho-q_{i}\right)\right)$-Cartesian; the left-hand one also is, for arbitrary $z$, because we just proved it; and therefore the one in the middle is $\left(c+\Sigma\left(\rho-q_{i}\right)\right)$-Cartesian, by lemma 1.3. 
3.3 Corollary Let $G$ be $\rho$-analytic with excess $c$. Suppose that $V \in \mathcal{O}$ has a proper Morse function with critical points of index $\leq q$ only, where $q<\rho$. Then $r_{k}$ from $T_{k} G(V)$ to $T_{k-1} G(V)$ is $(c+k(\rho-q))$-connected, for $k \geq 0$.

Remark Note that 3.3 is suggested but not implied by 2.3 , since $\eta_{k-1}=r_{k} \eta_{k}$. Also 3.3 does not imply 2.3 since it only depends on the behavior of $G$ on $\bigcup \mathcal{O} k$.

Proof of 3.3 We identify $r_{k}: T_{k} G \rightarrow T_{k-1} G$ with $\eta_{k-1}: T_{k} G \mapsto T_{k-1}\left(T_{k} G\right)$ (using [15, 6.1]). Now 2.3 can be applied, with $T_{k} G$ in place of $G$, because $T_{k} G$ is $\rho$-analytic with excess $c$ according to 3.2 .

\section{Haefliger's Theory}

Here we explain how the calculus of embeddings contains the Haefliger theory of smooth embeddings in the metastable range (which Haefliger unfortunately calls the stable range). First we recall Haefliger's theory. With $M$ and $N$ as before, we can make a commutative diagram

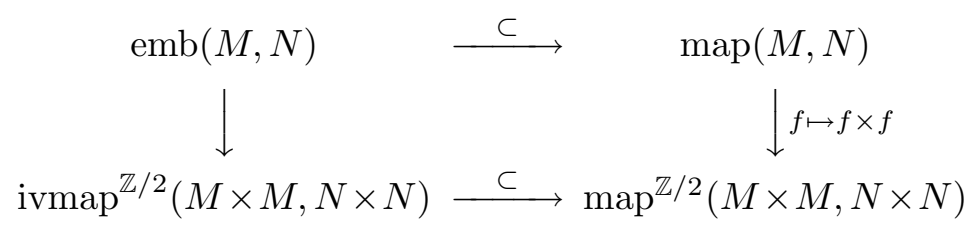

where all mapping Spaces in sight consist of smooth maps. Specifically, the expression $\operatorname{ivmap}^{\mathbb{Z} / 2}(\ldots)$ stands for a Space of strictly isovariant smooth maps, ie, equivariant smooth maps $g$ with the properties

$$
\begin{aligned}
g^{-1}\left(\Delta_{N}\right) & =\Delta_{M} \\
\left(T_{x} g\right)^{-1}\left(T_{g(x)} \Delta_{N}\right) & =T_{x} \Delta_{M} \quad \text { for any } x \in \Delta_{M} .
\end{aligned}
$$

(The second of these properties can be reformulated as follows: the vector bundle morphism induced by $g$, from the normal bundle of $\Delta_{M}$ in $M \times M$ to the normal bundle of $\Delta_{N}$ in $N \times N$, is a fiberwise monomorphism.)

4.1 Theorem [8] If $2 n>3(m+1)$ and $n \geq 3$, square (*) is 1-Cartesian.

Remark In Haefliger's set-up, the space corresponding to $\operatorname{ivmap}^{\mathbb{Z} / 2}(\ldots)$ consists of the (smooth or continuous) equivariant maps $g: M \times M \rightarrow N \times N$ for which $g^{-1}\left(\Delta_{N}\right)=\Delta_{M}$. The extra condition on infinitesimal behavior that we have added does affect the homotopy type. However, the comparison map is 1-connected (proved in [8] and especially [9]), so that 4.1 is correct with either definition. 
4.2 Lemma If $m \leq n$, the cofunctor $E$ taking $V \in \mathcal{O}$ to the homotopy pullback of

$$
\operatorname{ivmap}^{\mathbb{Z} / 2}(V \times V, N \times N) \stackrel{\subset}{\longrightarrow} \operatorname{map}^{\mathbb{Z} / 2}(V \times V, N \times N) \leftarrow \operatorname{map}(V, N)
$$

is polynomial of degree $\leq 2$.

Proof By $[15,2.5]$ it suffices to verify that each of the three terms from which the homotopy pullback is made is polynomial of degree $\leq 2$, as a cofunctor in $V$. Clearly $V \mapsto \operatorname{map}(V, N)$ is polynomial of degree $\leq 1$. The remaining two terms can be handled as in $[15,2.4]$. We omit the details.

4.3 Corollary Let $F(V)=\operatorname{emb}(V, N)$. The canonical morphism $F \rightarrow E$ is a second order Taylor approximation (induces an equivalence $T_{2} F \rightarrow T_{2} E$ ).

Proof By $[15,5.1]$ and 4.2 above it suffices to verify that $F(V) \rightarrow E(V)$ is a homotopy equivalence whenever $V$ is diffeomorphic to a disjoint union of $\leq 2$ copies of $\mathbb{R}^{m}$. But this is rather obvious.

We see that Haefliger's theorem, 4.1, can also be deduced from 4.3 and 2.5. In fact, 2.5 tells us that $F(M) \rightarrow E(M) \simeq T_{2} F(M)$ is $s$-connected with

$$
s=2(n-2-m)-m+1=2 n-3(m+1) .
$$

Again, this is not a new result. It is a reformulation of the main result of Dax's thesis [2].

\section{An Application}

The convergence statements 2.5 and 4.4 can be generalized mildly so that certain cases "with boundary" are included. What we have in mind is example 10.1 of [15]. Suppose therefore that $M^{m}$ and $N^{n}$ are smooth manifolds with boundary, $m \leq n$, and that a smooth embedding $g: \partial M \rightarrow \partial N$ has been selected. Let $\mathcal{O}$ be the poset of open subsets of $M$ containing $\partial M$. For $V \in \mathcal{O}$ let $\operatorname{emb}(V, N)$ be the Space of (neat and smooth) embeddings $V \rightarrow N$ which agree with $g$ near $V \cap \partial M$. The cofunctor $V \mapsto \operatorname{emb}(V, N)$ on $\mathcal{O}$ is good. A calculus of good cofunctors from $\mathcal{O}$ to Spaces is outlined in section 10 of [15].

5.1 Fact Let $F(V)=\operatorname{emb}(V, N)$ for $V \in \mathcal{O}$. If $m<n-2$, then $F(V) \simeq$ $\operatorname{holim}_{k} T_{k} F(V)$ for all $V$. In general, $F(V) \simeq \operatorname{holim}_{k} T_{k} F(V)$ provided $V$ has a handle decomposition relative to a collar on $\partial V$, with possibly infinitely many handles, all of index $\leq q$, where $q<n-2$. In this case $\eta_{k}: F(V) \rightarrow T_{k} F(V)$ is $(k(n-2-q)-q+1)$-connected. 
The proof is essentially identical with that of 2.5 . We omit it and turn to some examples. Suppose that $M$ is the unit interval $I$, and suppose for simplicity that it comes with a preferred smooth and neat embedding $I \rightarrow N$ (which we treat as an inclusion). Conditions on $N^{n}$ will be added later; for now, the only condition is $n \geq 4$. Then $V \mapsto \operatorname{emb}(V, N)$ is a functor from $\mathcal{O}$ to based Spaces. Notation: $\iota X=X \backslash \partial X$ for a manifold with boundary $X$.

Define $F(V)$ as in 5.1, for open $V \subset I$ containing the boundary. Our first and most important task is to understand the classifying fibration $p_{k}$ for $L_{k} F$, assuming $k \geq 2$. Its base space is

$$
\left(\begin{array}{c}
\iota I \\
k
\end{array}\right) \cong \mathbb{R}^{k}
$$

Its fiber over some $S$ (subset of $\iota I$ with $k$ elements) is the total homotopy fiber of the $k$-cube of pointed spaces

$$
R \mapsto \operatorname{emb}(R, \iota N) \quad(R \subset S) .
$$

Let $x \in S$ be the minimal element. In 5.2 and 5.3 below, $x \in S \subset I \subset N$ serves as base point of $\iota N$.

5.2 Lemma The total homotopy fiber of the $k$-cube $(*)$ is homotopy equivalent to the total homotopy fiber of the $(k-1)$-cube of pointed spaces

$$
R \mapsto \iota N \backslash R \quad(R \subset S, x \notin R) .
$$

Proof The idea is to use a Fubini principle: The total homotopy fiber of the $k$-cube $(*)$ is homotopy equivalent to the total homotopy fiber of the $(k-1)$ cube

$$
R \mapsto \text { hofiber }[\operatorname{emb}(R \cup x, \iota N) \rightarrow \operatorname{emb}(R, \iota N)] \quad(R \subset S, x \notin R) .
$$

The restriction map $\operatorname{emb}(R \cup x, \iota N) \rightarrow \operatorname{emb}(R, \iota N)$ is a fibration, so that its homotopy fiber can be replaced by its fiber (over the base point), which is $\iota N \backslash R$. Note that $R \subset S \subset \iota I \subset \iota N$.

5.3 Corollary The total homotopy fiber of the cube $(*)$ is homotopy equivalent to the total homotopy fiber of a cube of the form

$$
R \mapsto \iota N \vee\left(R_{+} \wedge \mathbb{S}^{n-1}\right) \quad(R \subset S \backslash x)
$$

Remark The cube in 5.3 is contravariant like $(*)$, ie, the maps in it are collapsing maps, not inclusion maps. The base point of $\iota N$ is still $x$. 
Proof Choose a smooth embedding $e: \iota N \rightarrow \iota N$, isotopic to the identity, such that $e(\iota N)$ and $S \backslash x$ are disjoint and $e(x)=x$. For each $y \in S \backslash x$ choose a smooth embedding $f_{y}: \mathbb{D}^{n} \rightarrow \iota N$ which maps the base point $(1,0, \ldots, 0)$ to $x$ and the center $(0, \ldots, 0)$ to $y$, and avoids all other points of $S$. Then for each $R \subset S \backslash x$, the map

$$
\iota N \vee\left(R_{+} \wedge \mathbb{S}^{n-1}\right) \vee\left((S \backslash R \backslash x)_{+} \wedge \mathbb{D}^{n}\right) \longrightarrow \iota N \backslash R
$$

which is $e$ on $\iota N$ and $f_{y}$ on $\{y\}_{+} \wedge \mathbb{S}^{n-1}$ and on $\{y\}_{+} \wedge \mathbb{D}^{n}$ (where applicable) is a homotopy equivalence. It is also contravariantly natural in the variable $R$. Further, for each $R \subset S \backslash x$, the collapse map

$$
\iota N \vee\left(R_{+} \wedge \mathbb{S}^{n-1}\right) \vee\left((S \backslash R \backslash x)_{+} \wedge \mathbb{D}^{n}\right) \longrightarrow \iota N \vee\left(R_{+} \wedge \mathbb{S}^{n-1}\right)
$$

is a homotopy equivalence, again contravariantly natural in the variable $R$. We have now reduced 5.3 to 5.2 .

In certain cases the Hilton-Milnor theorem [11] can be used to simplify 5.3 further. See [16] for the meaning of basic words, which we use in 5.4 below. For a basic word $w$ in the letters $z_{1}, \ldots, z_{k}$ we let $\alpha(w)$ be the number of letters distinct from $z_{1}$, and $\beta(w)$ the number of letters equal to $z_{1}$. For a pointed CW-space $Y$ the smash product $Y \wedge Y \cdots \wedge Y$ with $j$ factors will be abbreviated $Y^{(j)}$; the convention for $j=0$ is $Y^{(0)}=\mathbb{S}^{0}$.

5.4 Corollary Suppose that $N \simeq \Sigma Y$, where $Y$ is a connected pointed $C W$ space. Then the loop space of the total homotopy fiber of $(*)$ is homotopy equivalent to a weak product (union of finite products)

$$
\prod_{w}^{\prime} \Omega \Sigma^{1+\alpha(w)(n-2)} Y^{(\beta(w))}
$$

taken over all basic words $w$ in the letters $z_{1}, \ldots, z_{k}$ which involve all the letters except possibly $z_{1}$.

Before giving the proof, we must restate the Hilton-Milnor theorem. Let $X_{1}, \ldots, X_{k}$ be connected pointed CW-spaces. For a basic word $w$ in the letters $z_{1}, \ldots, z_{k}$ denote by $w\left(X_{1}, \ldots, X_{k}\right)$ the space obtained by substituting $X_{i}$ for $z_{i}$ and $\wedge$ for multiplication; for example, if $k=3$ and $w=\left(z_{2} z_{1}\right) z_{1}$, then $w\left(X_{1}, X_{2}, X_{3}\right)$ is $\left(X_{2} \wedge X_{1}\right) \wedge X_{1}$.

5.5 Hilton-Milnor Theorem $\Omega \Sigma\left(X_{1} \vee \cdots \vee X_{k}\right) \simeq \prod_{w}^{\prime} \Omega \Sigma\left(w\left(X_{1}, \ldots, X_{k}\right)\right)$, a weak product taken over the basic words $w$ in the letters $z_{1}, \ldots, z_{k}$. 
Proof of 5.4 We identify $S$ in 5.3 and 5.4 with $\{1,2, \ldots, k\}$, noting that $S$ has a preferred ordering since it is a subset of $I$. Fix $X_{1}=Y$ as in 5.4, and for $i>1$ regard $X_{i}$ as a variable with only two possible values, $\mathbb{S}^{n-2}$ and $*$. Letting the $X_{i}$ vary with these constraints, we find that

$$
\Omega \Sigma\left(X_{1} \vee \cdots \vee X_{k}\right)
$$

runs through the loop spaces of the vertices of the cube in 5.3. Each of these loop spaces can therefore be decomposed according to Hilton-Milnor. Moreover, the decomposition given by Hilton-Milnor is natural, a point which is stressed in [16], and we conclude that $\Omega$ of the cube in 5.3 splits into many separate factors indexed by the basic words $w$ in the letters $z_{1} \ldots, z_{k}$. Each factor is a $(k-1)$-cube in its own right, of the form

(**) $\quad R \mapsto \Omega \Sigma\left(w\left(Y, X_{2}(R), \ldots, X_{k}(R)\right) \quad(R \subset\{2, \ldots, k\})\right.$

where $X_{i}(R)=\mathbb{S}^{n-2}$ if $i \in R$ and $X_{i}(R)=*$ otherwise. If $w$ omits one of the letters $z_{2}, \ldots, z_{k}$, then the total homotopy fiber of $(* *)$ is contractible (by the Fubini priciple, also used in the proof of 5.2). Otherwise, the cube (**) has only one nontrivial vertex (the initial one) and its total homotopy fiber is therefore that vertex.

5.6 Corollary Suppose again that $N \simeq \Sigma Y$ as in 5.4. The terms of the Taylor tower of $\operatorname{emb}(I, N)$ are as follows, for $k \geq 2$ :

$$
L_{k} \operatorname{emb}(I, N) \simeq \prod_{w}^{\prime} \Omega^{k} \Sigma^{1+\alpha(w)(n-2)} Y^{(\beta(w))}
$$

where the weak product is over all basic words $w$ in the letters $z_{1}, \ldots, z_{k}$ which involve all the letters except possibly $z_{1}$.

Proof The $k$-th term of the Taylor tower can be described as the space of sections with compact support of a fibration $p_{k}$. See [15, section 10]. We found that the base space of $p_{k}$ is homeomorphic to $\mathbb{R}^{k}$, so that the section space in question is the $k$-fold loop space of any of the fibers. The fibers are described in 5.3, and their loop spaces in 5.4. Add a prefix $\Omega^{k-1}$ to both sides of the formula in 5.4.

5.7 Summary Suppose that $N^{n} \simeq \Sigma Y$ for a connected pointed $C W$-space $Y$, and $n \geq 4$, and $I$ is neatly embedded in $N$. Then $\operatorname{emb}(I, N)$ is homotopy equivalent to the homotopy inverse limit of a certain diagram

$$
\cdots \rightarrow T_{k} \operatorname{emb}(I, N) \rightarrow T_{k-1} \operatorname{emb}(I, N) \rightarrow \ldots T_{1} \operatorname{emb}(I, N) .
$$


Here $T_{1} \operatorname{emb}(I, N)$ is $\operatorname{imm}(I, N)$, the Space of smooth immersions which agree with the inclusion near $\partial I$. For $k \geq 2$, the homotopy fiber of the forgetful map $T_{k} \operatorname{emb}(I, N) \rightarrow T_{k-1} \operatorname{emb}(I, N)$ is homotopy equivalent to a weak product

$$
\prod_{w}^{\prime} \Omega^{k} \Sigma^{1+\alpha(w)(n-2)} Y^{(\beta(w))}
$$

taken over all basic words $w$ in the letters $z_{1}, \ldots, z_{k}$ which involve all the letters except possibly $z_{1}$.

5.8 Remarks In 5.7, let $N=\mathbb{R}^{n-1} \times I$. There is a distinguished neat embedding $I \rightarrow \mathbb{R}^{n-1} \times I$ which identifies $I$ with $0 \times I$. There is also a fibration sequence up to homotopy

$$
\operatorname{emb}\left(I, \mathbb{R}^{n-1} \times I\right) \longrightarrow \operatorname{emb}\left(\mathbb{S}^{1}, \mathbb{S}^{n}\right) \stackrel{f}{\longrightarrow} \mathrm{O}(n+1) / \mathrm{O}(n-1) .
$$

The arrow $f$ takes a smooth embedding $e: \mathbb{S}^{1} \rightarrow \mathbb{S}^{n}$ to the orthonormal 2frame in $\mathbb{R}^{n+1}$ consisting of $e(*)$ and the unit tangent vector to $e\left(\mathbb{S}^{1}\right)$ at $e(*)$. Consequently 5.7 calculates $\operatorname{emb}\left(\mathbb{S}^{1}, \mathbb{S}^{n}\right)$ if $n \geq 4$, up to extension problems in the homotopy category. We do not know what the extensions are, but we do hope that homotopy theorists will be intrigued.

Unfortunately our convergence result does not cover the approximation map from $\operatorname{emb}\left(I, \mathbb{R}^{2} \times I\right)$ to $\operatorname{holim}_{k} T_{k} \operatorname{emb}\left(I, \mathbb{R}^{2} \times I\right)$. With or without convergence, we get a map of component sets

$$
\pi_{0} \operatorname{emb}\left(I, \mathbb{R}^{2} \times I\right) \rightarrow \lim _{k} \pi_{0} T_{k} \operatorname{emb}\left(I, \mathbb{R}^{2} \times I\right) .
$$

It is generally believed [1] that this is closely related to Vassiliev's theory of knot invariants [12], [13]. Conversely, Kontsevich [10] used ideas related to Vassiliev's theory to prove results similar to 5.7, more specifically, to set up a spectral sequence converging to the rational cohomology of emb $\left(\mathbb{S}^{1}, \mathbb{R}^{n}\right)$ for $n \geq 4$. He was able to show that the spectral sequence collapses at the $E_{2}$ term. One hopes that this result can be reformulated and perhaps even re-proved in calculus language.

Acknowledgments It is a pleasure to thank John Klein for discussions related to the material in section 5 and for pointing out an error in an earlier version of section 2 .

The authors are partially supported by the NSF. 


\section{References}

[1] R Bott, On invariants of manifolds, from: "Modern methods in complex analysis", Ann. of Math. Stud. 137, Princeton Univ. Press, Princeton, NJ (1995) 29-39

[2] J-P Dax, Etude homotopique des espaces de plongements, Ann. Scient. de l'Ecole Norm. Sup. 5 (1972) 303-377

[3] T G Goodwillie, Calculus II: Analytic Functors, K-Theory, 5 (1991/1992) 295-332

[4] T G Goodwillie, A multiple disjunction lemma for smooth concordance embeddings, Amer. Math. Soc. Memoirs, 86 no. 431 (1990)

[5] T G Goodwillie, Excision estimates for spaces of homotopy equivalences, preprint, Brown University (1995)

[6] T G Goodwillie, Excision estimates for spaces of smooth embeddings, preprint, Brown University 1998

[7] T G Goodwillie, J Klein, Excision estimates for spaces of Poincaré embeddings, in preparation

[8] A Haefliger, Plongements différentiables dans le domaine stable, Commentarii Math. Helv. 37 (1962/63) 155-167

[9] A Haefliger, M Hirsch, Immersions in the stable range, Ann. of Math. 75 (1962) 231-241

[10] M Kontsevich, Feynman Diagrams and Low-dimensional Topology, from: "Proceedings of First European Congress of Mathematics (1992), vol. II" Birkhäuser, 97-121

[11] J Milnor, On the construction FK, from: "Algebraic Topology - a student's guide", by J F Adams, London Math. Soc. Lecture Note Series no. 4, Cambridge University Press (1972)

[12] V A Vassiliev, Cohomology of knot spaces, from: "Theory of singularities and its applications", (V I Arnold, editor), Advances in Soviet Mathematics (AMS) 1 (1990) 23-69

[13] V A Vassiliev, Complements to discriminants of smooth maps: Topology and Applications, Amer. Math. Soc. Press (1992)

[14] M Weiss, Calculus of embeddings, Bull. Amer. Math. Soc. 33 (1996) 177-187

[15] M Weiss, Embeddings from the point of view of immersion theory, Part I, Geometry and Topology, 3 (1999) 67-101

[16] G W Whitehead, Elements of Homotopy theory, Graduate texts in Mathematics, Springer (1978) 